\title{
Recent results on topology on the lattice (in memory of Pierre van Baal)
}

\author{
M. Müller-Preussker* \\ Humboldt-Universität zu Berlin, Institut für Physik, Newtonstr. 15, 12489 Berlin \\ E-mail: mmp@physik.hu-berlin.de
}

Memorizing Pierre van Baal we will shortly review his life and his scientific achievements. Starting then with some basics in gauge field topology we mainly will discuss recent efforts in determining the topological susceptibility in lattice QCD.

Pierre van Baal (Naarden, June 9, 1955 - Leiden, December 29, 2013) was a great theoretician many members of the lattice field theory community remember very well. He passed away much too early. He was not a 'latticist' by himself, but strongly interested in what one can learn from lattice theory about fundamental aspects of non-Abelian gauge theories like gluon and quark confinement. There are several colleagues who were inspired by his ideas and approaches. The author of this contribution and some of his coauthors are grateful to belong to them.

Pierre started his career with the B.Sc. in Physics and Mathematics in Utrecht. Having received his M.Sc. in 1980 he continued with the Ph.D. in Theoretical Physics at Utrecht University, where his advisor was Gerard 't Hooft. After that in 1984 he moved to Stony Brook first as a Research Associate and then as a Fellow in the joint Math/Phys Program. From 1987 to 1989 he became a Fellow at the CERN Theory Group. In 1989 he was appointed KNAW-Fellow by the Royal Academy of Sciences at University of Utrecht, before he became a full professor in Field Theory and Particle Physics at Instituut-Lorentz for Theoretical Physics of the University of Leiden in 1992. There he was not only a very motivated researcher but also an engaged teacher, even with projects for school kids. We all liked him as a very nice, modest person and a good friend of many of us. Having been attacked by a serious stroke just after returning from LATTICE '05 in Dublin, he found strong forces to recover and to reestablish his ability to talk, to travel and even to give lectures. Unfortunately and tragically, his hope and efforts to come back to research work - as he liked it so much - failed. ${ }^{\dagger}$

But now we feel how much we all miss him.

The 32nd International Symposium on Lattice Field Theory

23-28 June, 2014

Columbia University New York, NY

\footnotetext{
*Speaker.

$\dagger$ Pierre's statement in his C.V. (see www.lorentz.leidenuniv.nl/research/vanbaal/DECEASED/HOME/cv.html) describes the situation: "I had a stroke (bleeding in the head) on the evening of July 31, 2005. As a consequence of this I have accepted that since December 1, 2007 I am demoted to $20 \%$ and April 1, 2010 to $10 \%$ of a professorship. I could still teach (in a modified format), but since October 2008 I can not do it anymore. I can give seminars (twice as slow), but doing research (something new) is too difficult."
} 

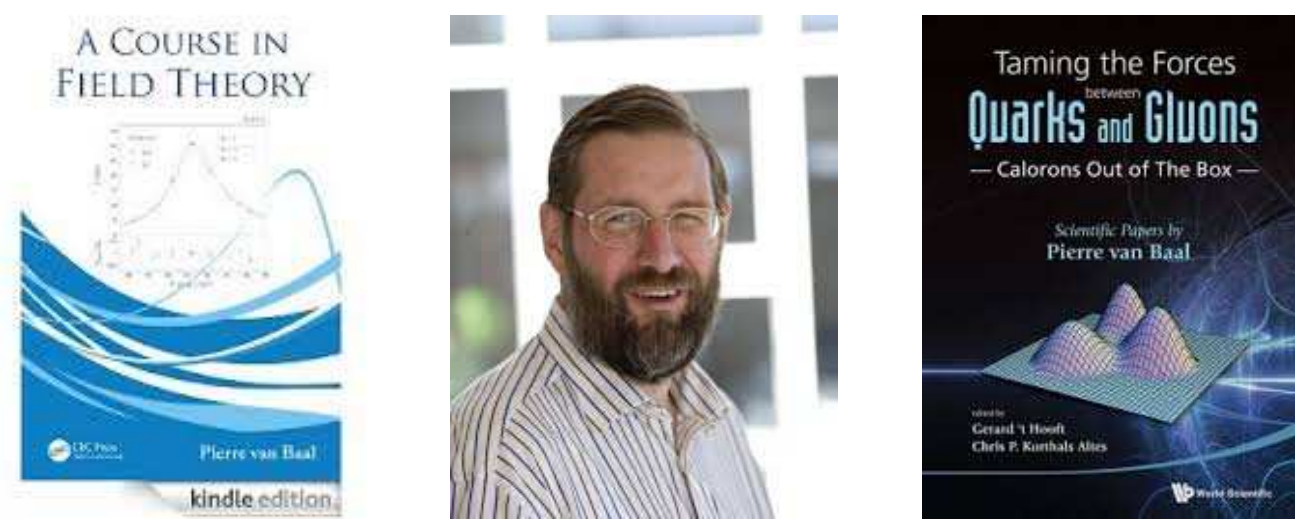

Figure 1: Pierre van Baal, his field theory lectures book [15] and his work collected by G. 't Hooft and C. Korthals Altes [16] celebrated at the Pierrefest in June 2013.

\section{Pierre van Baal's scientific achievements}

Pierre started his remarkable scientific career in theoretical physics by investigating $S U(N)$ gauge fields on a torus in particular with twisted boundary conditions [1]. Then he came to his "thoughts" on Gribov copies [2]. Searching for instantons from Monte Carlo generated lattice gauge fields he participated in inventing over-improved cooling [3] which is still in use, as we shall report later on. Thinking about improving lattice actions was a further lattice related matter of his work [4]. His thorough study of multi-instanton solutions and Nahm's transformation [5] about which several papers appeared over the years [6] led him together with his $\mathrm{PhD}$ student Thomas $\mathrm{C}$. Kraan and partly influenced by papers by Lee and Lu [7] to his probably most interesting invention with the strongest citation impact: periodic instantons (calorons) with nontrivial holonomy $[8,9]$. In the following years together with several young (and some senior) collaborators a series of papers appeared establishing various properties of those calorons which we want to call in the following KvBLL calorons $[10,11,12]$. Some reviews on KvBLL calorons and their relevance can be found in his and also other's talks $[13,14]$. Pierre's recommendable lectures on field theory were published in [15]. His work has been nicely collected in [16] (see Fig. 1).

\section{Topology, instantons, calorons - a 40 years old story}

Let us first recall some basic facts. Classical as well as path-integral determined quantum Euclidean Yang-Mills potentials $A_{\mu}(x)=A_{a, \mu}(x) T^{a} \in \operatorname{su}\left(N_{c}\right), \operatorname{tr}\left(T^{a} T^{b}\right)=\frac{1}{2} \delta^{a b}$, the properties of which with their field strength tensor $G_{\mu v}(x)$ are defined by the action

$$
S[A]=\frac{1}{2 g^{2}} \int d^{4} x \operatorname{tr}\left(G_{\mu v}(x) G_{\mu v}(x)\right),
$$

can be classified by a gauge invariant topological charge

$$
Q_{t}[A] \equiv \int d^{4} x \rho_{t}(x), \rho_{t}(x)=\frac{1}{16 \pi^{2}} \operatorname{tr}\left(G_{\mu v}(x) \tilde{G}_{\mu v}(x)\right), \quad \tilde{G}_{\mu \nu} \equiv \frac{1}{2} \varepsilon_{\mu v \rho \sigma} G_{\rho \sigma} .
$$

For finite-action fields in a volume $V \rightarrow \infty$ the topological charge $Q_{t}$ turns out to be integer-valued, because it can be expressed in terms of winding numbers or Pontryagin indices $w_{i}$ of continuous 
mappings of three-dimensional compact manifolds (surrounding possible singularities of the potentials at finite or infinite $x_{i}$ ) into the subgroup $S U(2)$, describing "homotopy classes" of the mapping $S^{(3)} \rightarrow S U(2) \equiv S^{(3)}$,

$$
Q_{t}[A] \equiv \sum_{i=1}^{q} w_{i} \in \mathbf{Z}
$$

The functional $Q_{t}[A]$ is invariant under continuous deformations, which for lattice discretized fields holds only if certain smoothness conditions are satisfied. From $\int d^{4} x \operatorname{tr}\left[\left(G_{\mu \nu} \pm \tilde{G}_{\mu \nu}\right)^{2}\right] \geq 0$ one immediately finds the continuum action $S[A]$ to be bounded from below in each topological sector

$$
S[A] \geq \frac{8 \pi^{2}}{g^{2}}\left|Q_{t}[A]\right| .
$$

Gauge field topology became a fundamentally interesting topic for QCD studies almost 40 years ago, when classical, topologically non-trivial field configurations, called BPST instantons [17], were found by solving the (anti)selfduality equation $G_{\mu v}= \pm \tilde{G}_{\mu v}$. The simplest $S U(2)$ solution (with $\frac{g^{2}}{8 \pi^{2}} S=\left|Q_{t}\right|=1$ ) in the singular gauge reads (with 't Hooft's symbols $\eta_{a \mu v}^{( \pm)}=\varepsilon_{a \mu v}$ for $\left.\mu, v=1,2,3, \eta_{a 4 v}^{( \pm)}=-\eta_{a v 4}^{( \pm)}= \pm \delta_{a v}, \eta_{a 44}^{( \pm)}=0\right)$

$$
A_{\mu}^{a(\mathrm{BPST})}(x)=R^{a \alpha} \eta_{\alpha \mu v}^{( \pm)} \frac{2 \rho^{2}(x-z)_{v}}{(x-z)^{2}\left((x-z)^{2}+\rho^{2}\right)}
$$

depending on eight modular space coordinates (position $z$, scale-size $\rho$, and global group space rotation $R$ ). Note that $S U\left(N_{c}\right)$ solutions are obtained by embedding $S U(2)$ solutions. The singular gauge instanton potential falls off as $x^{-3}$ at large $x$. Thus, the Yang-Mills path integral $\int D A \exp -S[A]$ can be semiclassically "approximated" by all possible superpositions of (anti) instantons sufficiently distant from each other. Evaluating the integral over leading order quantum flucuations around the (anti)instanton configurations, the path integral can be reduced to a partition function in the modular space of the instanton parameters [18]. This idea led from the dilute instanton gas model to an infrared regularized statistical mechanics of an instanton liquid [19].

Taking into account $N_{f}$ fermion flavor degrees of freedom $\psi_{f}$ with identical mass $m$, the effect of topologically non-trivial configurations like instantons enters through the axial anomaly [20]

$$
\partial_{\mu} j^{\mu 5}(x)=2 m P(x)+2 N_{f} \rho_{t}(x)
$$

with the topological charge density $\rho_{t}(x)$ according to Eq. (2.2) and

$$
j^{\mu 5}(x)=\sum_{f=1}^{N_{f}} \bar{\psi}_{f}(x) \gamma^{\mu} \gamma^{5} \psi_{f}(x), \quad P(x)=\sum_{f=1}^{N_{f}} \bar{\psi}_{f}(x) \gamma^{5} \psi_{f}(x) .
$$

By integrating Eq. (2.6) one gets a relation known as Atiyah-Singer index theorem [21]

$$
Q_{t}[A]=n_{+}-n_{-} \in \mathbf{Z}
$$

where $n_{+}\left(n_{-}\right)$is the number of zero modes of the massless Dirac operator $\gamma^{\mu} D_{\mu}[A]$ with positive (negative) chirality on the gauge field background $A$. A combination of the related Ward identities 
reads as an identity for the topological susceptibility [22]

$$
\begin{aligned}
\left.\chi_{t} \equiv \frac{1}{V}\left\langle Q_{t}^{2}\right\rangle\right|_{N_{f}} \equiv \int d^{4} x\left\langle\rho_{t}(x) \rho_{t}(0)\right\rangle & =-\frac{4 m}{\left(2 N_{f}\right)^{2}}\left\langle\sum_{f} \bar{\psi}_{f} \psi_{f}\right\rangle+\frac{(2 m)^{2}}{\left(2 N_{f}\right)^{2}} \int d^{4} x\langle P(x) P(0)\rangle \\
& =\frac{1}{2 N_{f}} m_{\pi}^{2} F_{\pi}^{2}+O\left(m_{\pi}^{4}\right),
\end{aligned}
$$

i.e. in full QCD it has to vanish linearly with $m_{\pi}^{2}$ in the chiral limit. As we shall see below, confirming this limit is still a challenge for lattice QCD. Note that Eq. (2.9) holds also on the lattice for Ginsparg-Wilson fermions [23, 24] (see below). Applying a $1 / N_{c}$ expansion, where fermion loop contributions become fully suppressed (quenched approximation, i.e. $N_{f}=0$ ), E. Witten (on the basis of current algebra theorems [25]) and G. Veneziano (taking the phenomenological spectrum into account [26]) have proposed the relation

$$
\chi_{t}^{\text {quen }}=\left.\frac{1}{V}\left\langle Q_{t}^{2}\right\rangle\right|_{N_{f}=0}=\frac{1}{2 N_{f}} F_{\pi}^{2}\left[m_{\eta^{\prime}}^{2}+m_{\eta}^{2}-2 m_{K}^{2}\right] \simeq(180 \mathrm{MeV})^{4} .
$$

Therefore, the existence of topologically non-trivial contributions to the path integral leads to the solution of the so-called $U_{A}(1)$ problem explaining that the $\eta^{\prime}$ meson (of the pseudoscalar flavor singlet current) is not a Goldstone boson in the chiral limit and why in nature $m_{\eta^{\prime}} \gg m_{\pi}$.

At this place it is worth to note, that the instanton liquid model of the QCD ground state describes reasonably well phenomena related to chiral symmetry and $U_{A}(1)$ symmetry breaking. However, without considering (still unkown) long-range correlations it fails to explain confinement. For more information see reviews of instanton physics by T. Schäfer and E. Shuryak [27] as well as by D. Diakonov [28], who has passed away also too early.

Let us turn to the case of non-zero temperature $T$. The analogous semiclassical treatment of the Yang-Mills partition function has been formulated in [29] based on Harrington-Shepard (HS) caloron solutions, i.e. $x_{4}$-periodic instanton chains $(1 / T=b)[30]$

$$
A_{\mu}^{a(\mathrm{HS})}(x)=\eta_{a \mu \nu}^{( \pm)} \partial_{v} \log \Phi(x)
$$

with $\Phi(x)-1=\sum_{k \in \mathbf{Z}} \frac{\rho^{2}}{(\vec{x}-\vec{z})^{2}+\left(x_{4}-z_{4}-k b\right)^{2}}=\frac{\pi \rho^{2}}{b|\vec{x}-\vec{z}|} \frac{\sinh \left(\frac{2 \pi}{b}|\vec{x}-\vec{z}|\right)}{\cosh \left(\frac{2 \pi}{b}|\vec{x}-\vec{z}|\right)-\cos \left(\frac{2 \pi}{b}\left(x_{4}-z_{4}\right)\right)}$,

omitting a possible global $S U(2)$ rotation. The topological charge of this solution is

$$
Q_{t} \equiv \frac{1}{16 \pi^{2}} \int_{0}^{b} d x_{4} \int d^{3} x \rho_{t}(x)= \pm 1
$$

As for BPST instanton solutions it exhibits trivial asymptotic holonomy, i.e. the untraced Polyakov loop at spatial infinity becomes an element of the center $\mathbf{Z}(2)$ of $S U(2)$,

$$
\mathbf{P} \exp \left(i \int_{0}^{b} A_{4}\left(\vec{x}, x_{4}\right) d x_{4}\right) \stackrel{|\vec{x}| \rightarrow \infty}{=} \mathscr{P}_{\infty} \in \mathbf{Z}(2) \text {. }
$$

Today we know that the HS caloron is only a special case of the more general and more complicated $K v B L L$ caloron solution already mentioned above. The $S U\left(N_{c}\right)$ KvBLL caloron in general 

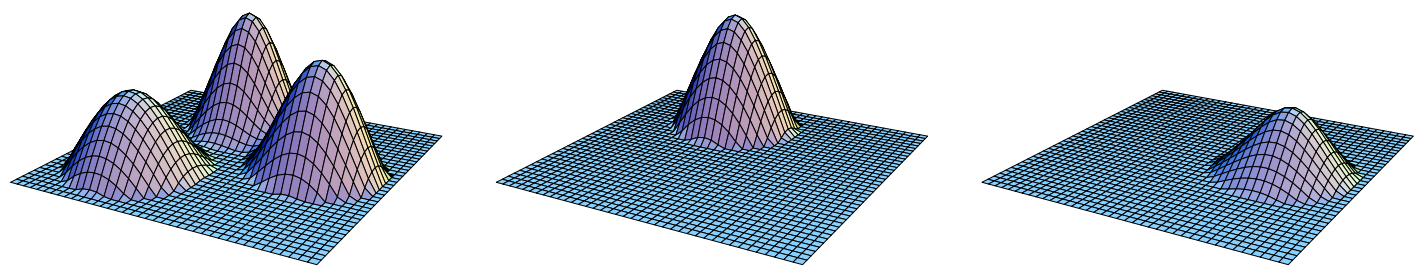

Figure 2: Left: Slice of the action density at $x_{4}=$ const . in a logarithmic scale for a single $S U(3) \mathrm{KvBLL}$ caloron with non-trivial holonomy showing three static monopole constituents well separated from each other. Middle (Right): Localisation of the zero mode for antiperiodic (periodic) boundary conditions for the fermion field in the $x_{4}$ direction. The figures are taken from Pierre van Baal's talk at JINR, Dubna in 1999 [13].

allows non-trivial holonomy, i.e. $\mathscr{P}_{\infty} \notin \mathbf{Z}\left(N_{c}\right)$. Such a caloron has $Q_{t}=1$ but consists of $N_{c}$ fractionally charged monopole constituents which turn into static (with respect to $x_{4}$ ) BPS monopoles, if the constituents are sufficiently separated from each other. Because of their selfduality these monopoles are often also called dyons. The action or topological charge of the latter are fully determined by the eigenvalues of the asymptotic holonomy $\mathscr{P}_{\infty}$ and constitute together the oneinstanton action $S_{\text {inst }}=8 \pi^{2} / g^{2}$ of the caloron. Fig. 2 shows a typical example for the $S U(3)$ case. It is obvious that such a configuration cannot be represented as a simple $S U(2)$ embedding. In the opposite limit, where the constituents are located near to each other, the action and the topological charge density of the KvBLL solution looks very similar to that of a HS caloron (or BPST instanton), i.e. concentrated within one lump of action and topological charge. Two further properties are characteristic for KvBLL solutions. First, the positions of the monopole or dyon constituents are given by those locations, where at least two eigenvalues of the local untraced Polyakov loop, i.e. the local holonomy, become degenerate [9]. Second, the zero mode of the massless Dirac operator in a KvBLL caloron background is localized only around one of the constituents (see the middle and right panels of Fig. 2). On which this happens depends on the boundary condition applied to the fermion field in the Euclidean time direction [10, 31].

These properties can be used as a trigger, when detecting calorons and/or dyons from MC generated thermal lattice gauge field configurations by cooling, 4D APE smearing or overlap operator mode expansions (see [32, 33, 34, 35] for $S U(2)$ pure gauge theory and more recently [36, 37] for $S U(3)$ ). The latter series of lattice investigations has led to a simple view of the topological structure of thermal Yang-Mills fields in terms of (anti)calorons and (anti)dyons. For $T<T_{c}$, where the spatially averaged Polyakov loop is fluctuating around zero, we see all possible dyon constituents with equal statistical weight, as one would expect them in a KvBLL caloron with maximally nontrivial (asymptotic) holonomy. For $T>T_{c}$, where the Polyakov loop average tends to $S U\left(N_{c}\right)$ center values and where one might expect caloron configurations with holonomies close to such values, topological clusters identifiable with corresponding heavy dyon constituents are found statistically suppressed. As a consequence, on one hand (anti)calorons - containing necessarily heavy and light (anti)dyons in this case - are rare, what explains the decreasing topological susceptibility with rising temperature. On the other hand, clusters, which can be interpreted (with the triggers 
mentioned above) as light dyons are abundant. This observation made for $S U(2)$ [34] as well as for $S U(3)$ [37] supports (non-Abelian) monopole dominance in the deconfinement phase.

Coming back to the continuum case, the dissociation of KvBLL calorons into dyon constituents has led to the hope to describe quark confinement at $T<T_{c}$ in terms of a liquid of correlated BPS monopoles or dyons. This would realize a picture of instanton quarks [38] studied many years ago in detail within the non-linear $O(3) \sigma$ model [39]. Work in such a direction strongly encouraged by Pierre van Baal was done over recent years mainly by three groups [40] (see also talk by E. Shuryak). It is worth mentioning that KvBLL calorons carry not only magnetic monopole world lines (seen on the lattice within the maximally Abelian gauge) but also extended center vortex structures [35], which seems to provide a bridge to confinement in terms of center vortices, too (see the reviews by J. Greensite [41]). Finally, it is worth mentioning that Pierre's work has influenced also a new systematic development of the semiclassical approach within perturbation theory called resurgent trans-series expansions [42] (cf. talk by M. Ünsal).

\section{How to measure topology on the lattice}

Evaluating the topological charge $Q_{t}$ and correspondingly the topological susceptibility $\chi_{t}$ as well as identifying topological excitations on the lattice are old issues but remain important challenges until today. There are two ways to address this question related to each other via the axial anomaly (2.6) or the index theorem (2.8). The first one expresses the topological charge directly by the lattice gluon field strength $G_{\mu \nu}$. This is easily done with a plaquette loop representation [43], but the lattice $Q_{t}$ is not an integer, and the corresponding topological susceptibility requires the subtraction of a perturbative tail and a proper renormalization [44]. In combination with various methods of stripping off quantum fluctuations i) by cooling - originally invented in order to extract approximate multi-instanton solutions [45], ii) by 4D APE [46], stout [47] or HYP smearing [48], iii) by (inverse) blocking, smoothing or cycling [49], or iv) by the gradient flow [50] one ends up with smooth lattice gauge field configurations, for which (improved) loop definitions provide $Q_{t}$ values being very close to integers. All these methods applied with a well-defined resolution scale allow to reveal the topological structure of the Monte Carlo generated lattice gauge fields in terms of clusters of topological charge.

Alternatives to determine $Q_{t}$ are given by geometric definitions of $Q_{t}$ which rely on the homotopy properties of the gauge field even on the lattice (with torodial boundary conditions). Such prescriptions were invented in the past by M. Lüscher [51], P. Woit [52] and A. Phillips and D. Stone [53]. They all provide integer values by definition. But due to lattice artifacts on rough lattice configurations they may yield different numbers. Only sufficiently smoothed fields will provide a unique answer. Sufficient conditions for such a smoothness exist and can be expressed in terms of upper bounds on the action density [51].

The second approach to determine $Q_{t}$ employs various fermionic definitions. The basic observation is that any lattice Dirac operator obeying the Ginsparg-Wilson relation [54]

$$
\gamma_{5} D+D \gamma_{5}=a D \gamma_{5} D
$$

satisfies the index theorem $(2.8)[55,56]$. Such Dirac operators have been realized within the perfect action approach [55], with Neuberger's overlap operator [57] as well as with domain wall 
fermions with an extra dimension [58]. But even for these constructions of Dirac operators holds that the topological charge given by their index is not uniquely defined due to lattice artifacts.

Similarly to applying the gluonic definitions in combination with some smoothing prescription one can use a fermionic filtering method by representing the topological charge density $\rho_{t}$ in terms of a finite set of low-lying modes of the choosen lattice Dirac operator $D$,

$$
\rho_{t}(x)=\operatorname{tr} \gamma_{5}\left(\frac{1}{2} D_{x, x}-1\right)=\sum_{n=1}^{N}\left(\frac{\lambda_{n}}{2}-1\right) \psi_{n}^{\dagger}(x) \gamma_{5} \psi_{n}(x) .
$$

Indeed, comparing this filtering prescription with APE or stout smearing has shown that the number of smearing steps can be optimized to a given number $N$ of low-lying modes such that the same local topological structures are seen in terms of scale dependent clustering of topological charge [59] and its varying fractal dimensionality [60]. One should expect that this holds also for other smoothing methods including also the Wilson or gradient flow. We shall come back to this point below (see Section 4.1).

Other possibilities to determine $Q_{t}$ are given in terms of the anti-Hermitean Dirac operator $\not D$ [61] or by counting the index from the spectral flow of the Hermitian Wilson-Dirac operator [62]. More recently a new fermionic method has been proposed by representing the topological susceptibility in terms of higher moments of scalar and pseudo-scalar currents and spectral projectors [24, 63, 64, 65, 66, 67], see Section 4.3.

\section{Selected recent lattice results}

\subsection{Cooling versus gradient (Wilson) flow}

The old days' cooling method used for the search for multi-instanton solutions [45] and more recently to establish the non-trivial holonomy KvBLL calorons $[68,32,12,36]$ solves the lattice field equations locally (for a given link variable), replaces the old by new link variable, steps through the lattice (while the order is not unique), and, if sufficiently often repeated, ends up at more or less stable plateau values for the topological charge and action. The method has much improved as over-improved cooling [3, 68, 69] stabilizing (multi)instantons or calorons and therefore providing extremely stable plateaus at nearly integer $Q_{t}$ values. With an improved lattice representation of the field strength tensor $G_{\mu \nu}$ [70] one can nicely check the degree of (anti)selfduality e.g. by comparing the topological charge with the action in instanton units.

Recent examples of typical cooling histories for gluodynamics at $T>0$ obtained on lattice sizes $16^{3} \times 4$ can be seen from Fig. 3. As we have argued in [71] the stability (decay) of plateaus for $T<T_{c}\left(T>T_{c}\right)$ can be traced back to the KvBLL caloron structure and to its non-trivial (trivial) asymptotic holonomy, i.e. to the dyon constituent mass symmetry (asymmetry). For $T>T_{c}$ (anti)selfdual plateaus were occuring very rarely. Searching for with respect to $\left|Q_{t}\right|$ first stable plateaus the topological susceptibility $\chi_{t}$ for gluodynamics as well as for full QCD with $N_{f}=2$ clover-improved Wilson fermions is obtained versus $T / T_{c}$ as shown in Fig. 4 (for details cf. [71]). It is obvious that $\chi_{t}$ behaves much smoother through the crossover of full QCD than passing the first order transition in gluodynamics. The behavior of the topological susceptibility at and beyond the transition is essential for understanding the mechanism of $U_{A}(1)$ restoration (and in the twoflavor case for determining the universality class of the transition in the limit $m_{\pi} \rightarrow 0$ ). It seems to 

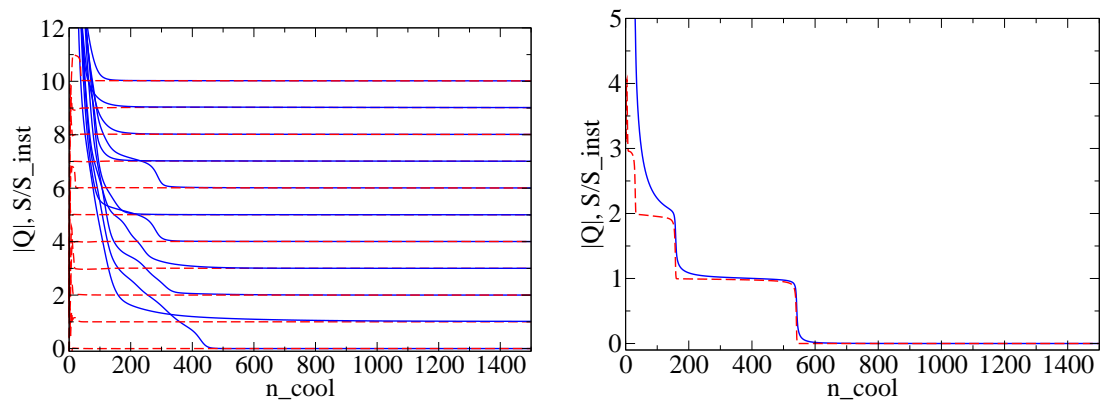

Figure 3: Cooling histories for pure gluodynamics at non-zero temperature (from [71]). We show the action in instanton units $S / S_{\text {inst }}$ (blue full lines) and the topological charge $Q_{t}$ (red dashed lines), both represented with an lattice-improved field strength tensor, see the text. Left: for confinement at $T=0.88 T_{c}$. Right: for deconfinement at $T=1.12 T_{c}$.
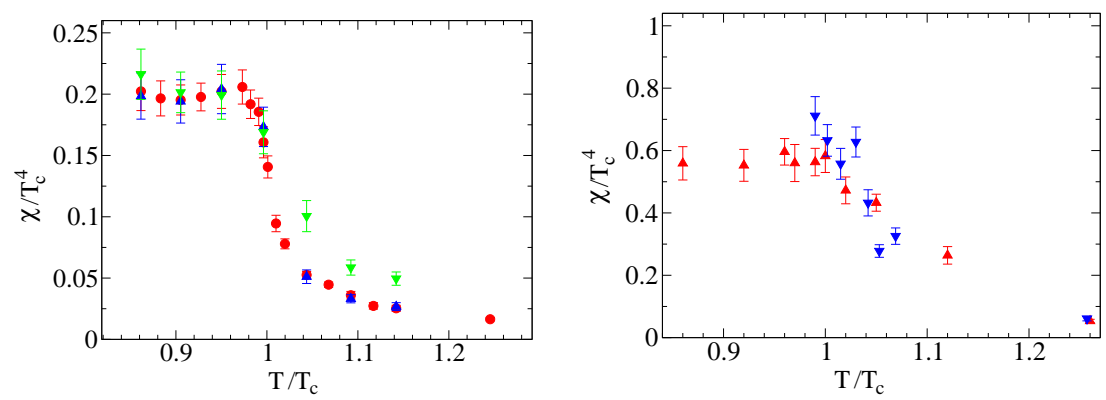

Figure 4: Topological susceptibility $\chi_{t}$ vs. $T / T_{c}$ obtained with over-improved cooling [71]. Left: for gluodynamics with lattice sizes $16^{3} * 4$ (red circles), $24^{3} * 4$ (blue up triangles) and $24^{3} * 6$ (green down triangles). Right: for full QCD with $N_{f}=2$ clover-improved Wilson fermions for lattice sizes $16^{3} * 8$ (red up triangles) and $24^{3} * 8$ (blue down triangles). The pion mass is of $O(1 \mathrm{GeV})$.

be too early to draw any final conclusion before taking the chiral limit in a proper way. Therefore, it may not wonder, that different groups having recently discussed the issue of $U_{A}(1)$ restoration still come to different conclusions [72].

Compared to (improved) cooling as discussed before, the gradient flow stands theoretically on a more sound basis. Proposed and thoroughly investigated by M. Lüscher since 2009 [50] and studied also with respect to perturbation theory [73] it provides an easy controllable manner to remove UV fluctuations (cf. his plenary talks at LATTICE 2010 and 2013 [74]). It's flow time evolution describing a diffusion process at scale $\lambda_{s} \simeq \sqrt{8 t}, t=a^{2} \tau$ and continuously minimizing the action is uniquely defined for an arbitrary lattice field $\left\{U_{\mu}(x)\right\}$ by solving

$$
\dot{V}_{\mu}(x, \tau)=-g_{0}^{2}\left[\partial_{x, \mu} S(V(\tau))\right] V_{\mu}(x, \tau), \quad V_{\mu}(x, 0)=U_{\mu}(x)
$$

The physical scale to stop the flow can be efficiently fixed by demanding e.g.

$$
\left.t^{2}\left\langle\frac{1}{2} \operatorname{tr} G_{\mu \nu} G_{\mu \nu}\right\rangle\right|_{t=t_{i}}=T_{i}, \quad i=0,1 \quad \text { with } \quad T_{0}=0.3, T_{1}=\frac{2}{3} .
$$


Simple renormalization properties, in particular in the fermionic sector, and the emergence of topological sectors at sufficient large diffusion scale are clear advantages of the method.

However, this does not mean that the previously mentioned cooling or smearing methods have to be abandoned. A comparison and mutual optimization of the fermionic filtering method with those pure gauge field methods have demonstrated a correspondence between them [59]. Indeed, the gradient flow can be mapped to cooling in the ensemble average, as C. Bonati and M. D'Elia have recently shown [75]. In the pure gluodynamic case with the standard Wilson plaquette action, for given numbers of cooling sweeps $n_{c}$ they have determined the Wilson flow time $\tau$, which - in the average - provides the same plaquette action. They have estimated the dimensionless flow time $\tau$ on a perturbative ground and found it well satisfied via numerical simulations to be $\tau=n_{c} / 3$ as can be seen from the left panel of Fig. 5. On the right panel of the same figure one nicely sees the
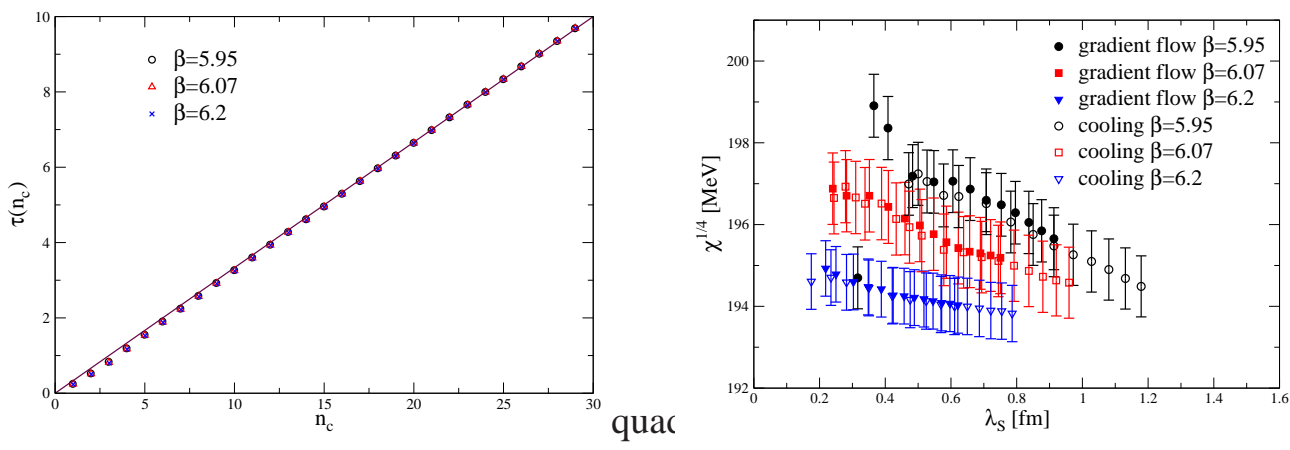

Figure 5: Left: Flow time $\tau$ vs. number of cooling steps $n_{c}$. Right: topological susceptibility $\chi_{t}^{\frac{1}{4}}$ vs. diffusion scale $\lambda_{s}$ for cooling and gradient (Wilson) flow at various lattice scales. Both figures taken from [75].

topological susceptibility for cooling and for the Wilson flow to agree completely. Moreover, the strong dependence on the lattice spacing at fixed diffusion scale $\lambda_{s}$ becomes obvious. Additionally, the authors convinced themselves that cooling and Wilson flow reveal the same local topological structure with high confidence. Let us add that recently also Wilson loops have been computed with smearing and gradient flow. They were found to agree to a high degree (see [76] and talk by M. Okawa).

\subsection{Exploring the mass dependence of $\chi_{t}$ in full QCD}

Only over the last years the expected chiral behavior of the topological susceptibility $\chi_{t} \sim$ $F_{\pi}^{2} m_{\pi}^{2} \sim m_{q}\langle\bar{q} q\rangle$ has found a real confirmation from lattice full QCD. Let us briefly sketch some recent work in this direction.

The SINP Kolkata group [77, 78] has employed the standard Wilson gauge and fermion action $\left(N_{f}=2\right)$ at $m_{\pi} \geq 300 \mathrm{MeV}$. The topological charge $Q_{t}$ was measured with the blocking-inverse blocking (smoothing) method [49]. An improved ansatz for the density $\rho_{t}$ was taken. The topological correlation function for varying volume and quark mass has been studied. A non-negligible lattice spacing effect could also be made transparent. From the left panel of Fig. 6 we observe a clear descent towards vanishing $\chi_{t}$ with a pion mass squared tending to zero, while on the right 

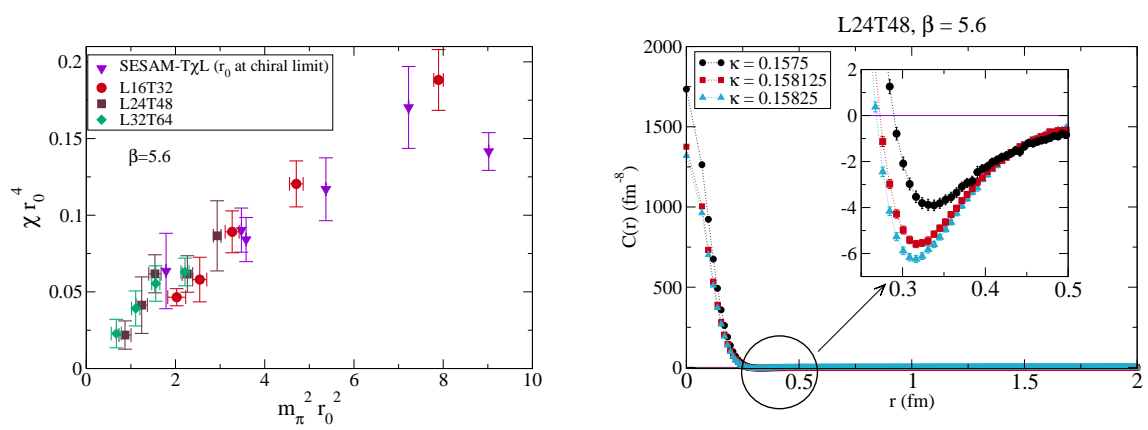

Figure 6: Left: $\chi_{t}$ versus $m_{\pi}^{2}$ for several volumes. For comparison older data from SESAM-T $\chi$ L collaboration [79] is shown (figure taken from [77]). Right: $\rho_{t}$ correlation function $C_{t}(r)$ at different quark mass values in terms of the Wilson hopping parameter $\kappa$ (from [78]).

panel the space correlation function $C_{t}(r)$ of the topological density shows the expected change in sign and a behavior becoming the steeper the smaller the quark mass is.

A brand-new topology Wilson flow analysis of the ALPHA collaboration has been presented at this conference by M. Bruno [80]. Since the Wilson flow can be stopped at a well-defined scale, the results should be under better control than the previously mentioned ones. The ALPHA collaborators studied $N_{f}=2$ lattice QCD with $O(a)$-improved Wilson fermions and standard Wilson gauge action. They investigated the Wilson flow on CLS ensembles with three lattice spacings, for $m_{\pi} \in[190,630] \mathrm{MeV}$ and a lattice extent $L m_{\pi}>4$. They employed periodic as well as open boundary conditions. For the author it came somewhat as a surprise that $Q_{t}$ autocorrelations were observed to become weaker with decreasing pion mass ${ }^{1}$. An overall fit to $\chi_{t}$ with a $\chi \mathrm{PT}$ ansatz like $t_{1}^{2} \chi_{t}=c t_{1} m_{\pi}^{2}+b \frac{a^{2}}{t_{1}}$ describes the mass dependence sufficiently well (with $t_{1}$ denoting the flow scale according to Eq. (4.2)). However, lattice artifacts turned out to be strong and a proper chiral limit only possible after the continuum extrapolation is taken (see Fig. 7). Compared to the quenched case also shown in the figure the full QCD result turns out to be quite strongly suppressed over the whole range of pion masses studied.

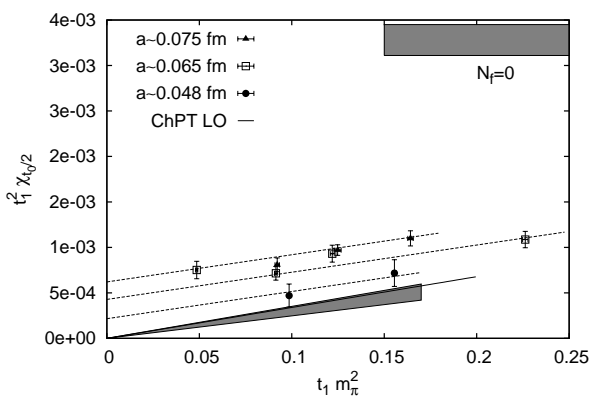

Figure 7: Wilson flow estimated topological suceptibility $\left.\chi_{t}\right|_{t=t_{0} / 2}$ versus $m_{\pi}^{2}$ - both in units of $t_{1}$-reported by the ALPHA collaboration [80].

Preliminary results of a gradient flow analysis for the topological susceptibility were reported

\footnotetext{
${ }^{1}$ The author thanks S. Mondal for the information that such an observation has been reported also in [81].
} 
by QCDSF (R. Horsley, G. Schierholz et al) for $N_{f}=2+1$ QCD with a tree-level Symanzik improved gauge action and (stout smeared) clover-improved Wilson fermions. In this investigation QCDSF follows two chiral limit strategies: i) $m_{u}=m_{d}=m_{s} \rightarrow 0$ and ii) $m_{u}=m_{d} \rightarrow 0$, while $m_{u}+m_{d}+m_{s}=\bar{m}=$ const. with $\bar{m}$ tuned to its physical value. The corresponding dependence of the topological susceptibility on the pion mass can be jointly fitted on the basis of the flavor-singlet and flavor-octet Gell-Mann-Oakes-Renner relations (see Fig. 8).

Let us conclude this discussion with the comment that the continuum limit for gradient flow estimates can be further improved (cf. talks by A. Ramos, S. Sint and D. Nogradi $[82,83]$ ).
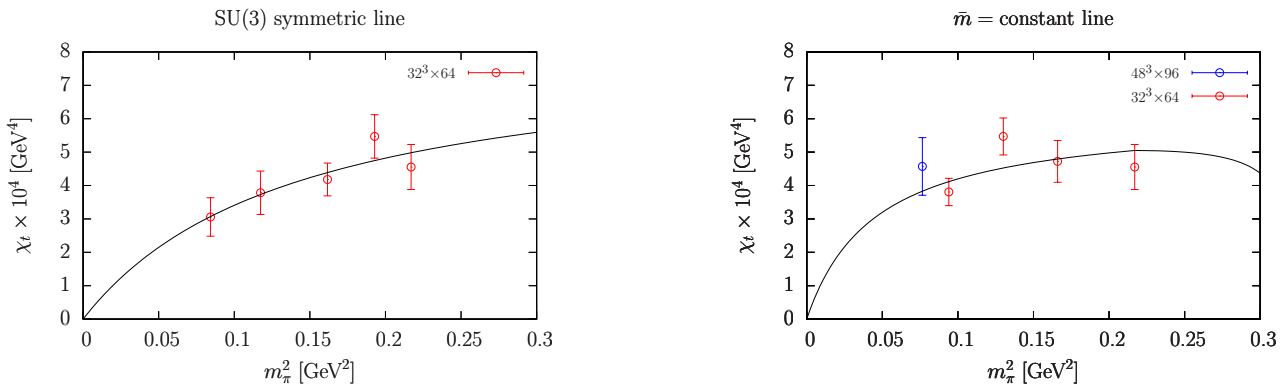

Figure 8: Topological susceptibility fixed at gradient flow scale $t_{0}$ versus $m_{\pi}^{2}$ for two chiral limit strategies as explained in the text (communicated by QCDSF, thanks to G. Schierholz).

\subsection{Spectral projector method applied to twisted mass fermions}

Ten years ago, extending an analysis presented in Ref. [24] M. Lüscher succeeded to propose a fermionic representation for the topological susceptibility $\chi_{t}$ in terms of singularity-free density chain correlators, which has not to be renormalized [63]. Treated with spectral projectors $\mathbf{P}_{M}$ allowing to project onto the subspace of $D^{\dagger} D$ eigenmodes below certain threshold $M^{2}$ and approximating them by rational functions $\mathbf{R}_{M}$ (see [64]) a first computation in pure gluodynamics became possible two years later [65]. For the valence quarks they used two-flavor clover-improved Wilson fermions. The numerical result for $\chi_{t}^{\text {quen }}$ turned out to be in good agreement with the corresponding result [84] from the index theorem studied with Neuberger's overlap operator [57] and also with the phenomenological value (see Eq. (2.10). To my knowledge, for the first time this approach has been applied to compute $\chi_{t}$ (and the chiral condensate) in full QCD by the ETM collaboration [66, 67]. The authors used dynamical Wilson twisted mass fermions with $N_{f}=2$ as well as $N_{f}=2+1+1$ flavor degrees of freedom (cf. talks by E. Garcia Ramos and K. Cichy). The topological susceptibility has been represented and approximated as

$$
\begin{aligned}
\chi_{t} & =\frac{1}{V} \frac{\left\langle\operatorname{Tr}\left\{\mathbf{R}_{M}^{4}\right\}\right\rangle}{\left\langle\operatorname{Tr}\left\{\gamma_{5} \mathbf{R}_{M}^{2} \gamma_{5} \mathbf{R}_{M}^{2}\right\}\right\rangle}\left\langle\operatorname{Tr}\left\{\gamma_{5} \mathbf{R}_{M}^{2}\right\} \operatorname{Tr}\left\{\gamma_{5} \mathbf{R}_{M}^{2}\right\}\right\rangle \\
& =\frac{1}{V} \frac{Z_{S}^{2}}{Z_{P}^{2}}\left\langle\operatorname{Tr}\left\{\gamma_{5} \mathbf{R}_{M}^{2}\right\} \operatorname{Tr}\left\{\gamma_{5} \mathbf{R}_{M}^{2}\right\}\right\rangle=\frac{1}{V} \frac{Z_{S}^{2}}{Z_{P}^{2}}\left(\left\langle\mathscr{C}^{2}\right\rangle-\frac{\langle\mathscr{B}\rangle}{N}\right),
\end{aligned}
$$

where $Z(2)$ random estimators have been used to estimate

$$
\mathscr{B}=\frac{1}{N} \sum_{k=1}^{N}\left(\mathbf{R}_{M} \gamma_{5} \mathbf{R}_{M} \eta_{k}, \mathbf{R}_{M} \gamma_{5} \mathbf{R}_{M} \eta_{k}\right) \text { and } \mathscr{C}=\frac{1}{N} \sum_{k=1}^{N}\left(\mathbf{R}_{M} \eta_{k}, \gamma_{5} \mathbf{R}_{M} \eta_{k}\right)
$$


Note that the renormalization constants satisfy $\frac{Z_{s}}{Z_{P}}=1$ and $\mathscr{C} \equiv Q_{t} \in \mathbb{Z}$ for $N \rightarrow \infty$, if $D$ is a Ginsparg-Wilson operator (e.g. the overlap operator), i.e. $\mathscr{C}$ plays conditionally the role of the topological charge. For the Wilson twisted mass discretization one can rely on automatic $O(a)$ improvement [85]. Since the authors used also an improved gauge action they could hope for a weak $a$-dependence of the topological susceptibility. The renormalization constants $Z_{S}, Z_{P}$ can be taken also from other ETMC evaluations [86]. The "topological" charge $\mathscr{C}$ turned out nicely Gaussian-like distributed. In Fig. 9 selected results are shown. The left panel demonstrates the projector method computation of the $Z$-factor ratio to be consistent with that of Refs. [86]. The right panel shows the topological susceptibility as a function of the quark mass. Within the error bars, which are still quite large, the different lattice scale results more or less agree, indeed. In any case the behavior of $\chi_{t}$ is seen to be compatible with a linear decrease with the quark mass towards the chiral limit. The slope of this curve allows also to determine the chiral condensate. The result of this estimate was consistent with that of other methods. Finally, in the quenched limit $\chi_{t}^{\text {quen }}$ came out in good agreement with Eq. (2.10) (cf. E. Garcia Ramos' talk).
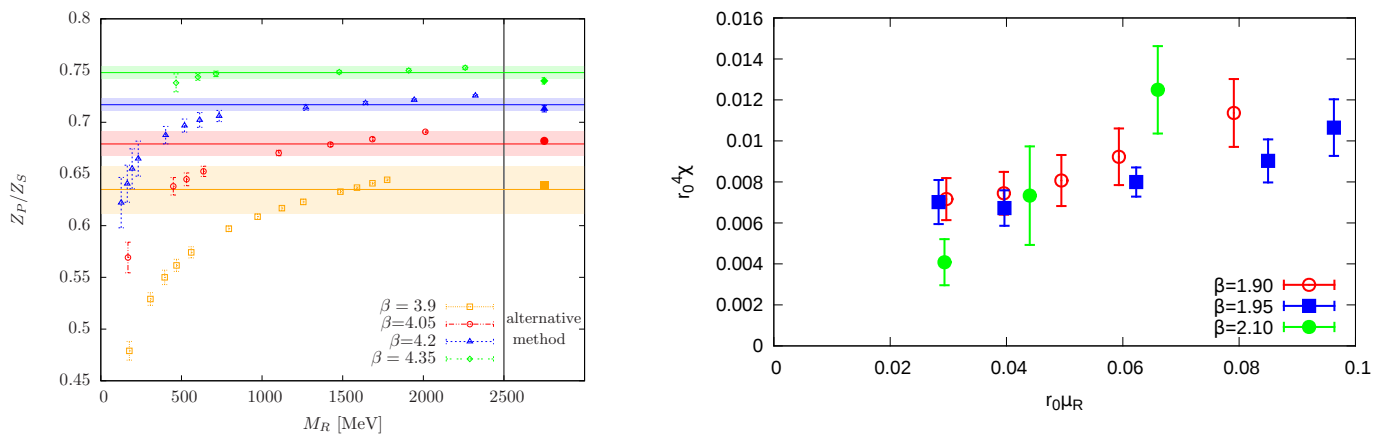

Figure 9: Left: The ratio of renormalization constants $\frac{Z_{S}}{Z_{P}}$ versus threshold mass $M_{R}$ for $N_{f}=2$. In the right most part of the panel results of alternative computations are shown [86]. Right: The $N_{f}=2+1+1$ result for $\chi_{t}$ in units of the Sommer scale $r_{0}$ [87] versus renormalized quark mass $\mu_{R}$ for three lattice spacings. Figures are taken from [67].

\subsection{Comparing various methods to determine $Q_{t}$ and $\chi_{t}$}

The ETM collaboration has made a joint effort [88] to compare various methods to compute the topological susceptibility within the framework of $N_{f}=2$ twisted mass fermions and tree-level Symanzik improved gauge action. All computations were done on the same set of configurations for a pion mass value $m_{\pi}=300 \mathrm{MeV}$, with a lattice spacing $a=.081 \mathrm{fm}$ and linear lattice size $L=$ $1.3 \mathrm{fm}$. Without taking the continuum limit one should not really expect a full agreement even in case the methods are really equivalent and correctly established. The authors compared fermionic definitions of the topological charge (index of the overlap operator, Wilson-Dirac operator spectral flow (SF), spectral projector method (SP)) with gluonic field strength (FT) definitions applying various versions and stages of gradient flow (GF), cooling, and APE/HYP smearing. In Fig. 10 we see how the different methods to determine $Q_{t}$ are correlated. On the right hand side the colored correlation scale from bottom (blue = weak correlation) to top (red=strong correlation) is given. Most of the versions are strongly correlated among each other, except the gluonic FT one without any removal of perturbative fluctuations. This is a well-known fact since the early days 
of topological lattice investigations. That also the spectral projector results are weakly correlated to the other ones does not come as a surprise, too, because of the stochastic determination of the 'charge' $\mathscr{C}$ in accordance with Eq. (4.4). A comparison of the resulting $\chi_{t}$ values shows that all methods provide results in the same ball park. This holds also for the spectral projector method, provided the spectral threshold $M_{R}$ is taken high enough. For details we refer to [88]. The outcome

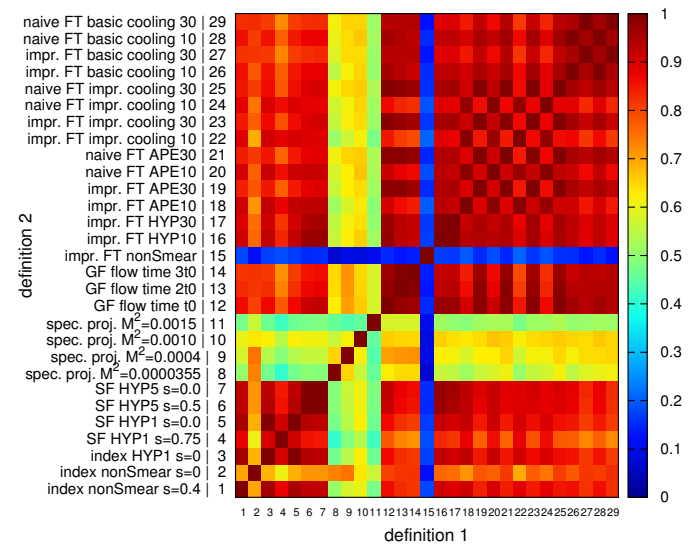

Figure 10: Correlation matrix between different methods to determine the topological charge (from [88]).

of the ETMC comparison certainly allows the conclusion that the well-controllable gradient flow method at the first place, but also cooling or smearing methods, if adapted with analogous criteria to fix the diffusion scale, are optimal (also from the computational point of view) in order to determine topological properties of lattice QCD.

\section{Conclusions}

First of all I have to apologize for not having discussed many issues, which have been touched during this symposium and would have been of interest here - as they would for Pierre van Baal. The following topics belong to the problems within the range of this short review but could not be covered here:

- the recent $\eta^{\prime}-\eta$ mixing results [89], which became possible due to various powerful noise reduction techniques [90],

- the $U_{A}(1)$ symmetry restoration puzzle mentioned already above [72],

- the use of open boundary conditions suppressing HMC's autocorrelation for $Q_{t}[91,80]$

(cf. talk by G. Mc Glynn),

- the simulation of $\theta$-vacua with Langevin techniques or dual variables

(talks by L. Bongiovanni, T. Kloiber),

- considerations with fixed topology (talks by A. Dromard, H. Fukaya, U. Gerber, J. Verbaarschot), - ongoing discussions about the vacuum structure and topological excitations (talks by N. Cundy, P. de Forcrand, M. Hasegawa, M. Ogilvie, A. Shibata, H.B. Thacker, D. Trewartha, M. Ünsal), - phase structure at differing $m_{u}, m_{d}$ masses ([92] and talk by S. Aoki),

- topology in related theories as $G_{2}$ Yang-Mills theory and $N=1$ SUSY on the lattice ([93] and talk by P. Giudice),

- effects in QCD caused by magnetic background fields [94]. 
Let us briefly summarize. Investigations of KvBLL caloron and dyon gas models with nontrivial holonomy as initiated by Pierre van Baal are still interesting and encouraging for better describing (de)confinement within the finite temperature setting of QCD. Even more, they may pave a way to improve systematically the semiclassical approach. The computation of the topological susceptibility with new methods (gradient flow, spectral projector method) is on a promising way. In any case one has to keep track of lattice artifacts and to study the continuum limit.

\section{Acknowledgments}

I would like to express my gratitude to the organizers for having been invited to give this talk. Many thanks to all those who have provided material to be reviewed, sorry to those I have not mentioned.

\section{Thank you, Pierre, your vision and ideas are alive.}

\section{References}

[1] P. van Baal, Commun.Math.Phys. 85 (1982) 529; Commun.Math.Phys. 94 (1984) 397.

P. van Baal and J. Koller, Annals Phys. 174 (1987) 299.

J. Koller and P. van Baal, Nucl.Phys. B302 (1988) 1.

[2] P. van Baal, Nucl.Phys. B369 (1992) 259.

[3] M. García Pérez, A. González-Arroyo, J. R. Snippe, and P. van Baal, Nucl.Phys. B413 (1994) 535, arXiv:hep-lat/9309009 [hep-lat].

[4] M. García Pérez, J. R. Snippe, and P. van Baal, Phys.Lett. B389 (1996) 112, arXiv:hep-lat/9608036 [hep-lat].

[5] M. Atiyah, N. J. Hitchin, V. Drinfeld, and Y. Manin, Phys.Lett. A65 (1978) 185. W. Nahm, Phys.Lett. B90 (1980) 413.

[6] P. J. Braam and P. van Baal, Commun.Math.Phys. 122 (1989) 267. P. van Baal, Phys.Lett. B448 (1999) 26, arXiv: hep-th/9811112 [hep-th] . M. García Pérez, A. González-Arroyo, C. Pena, and P. van Baal, Nucl.Phys. B564 (2000) 159, arXiv:hep-th/9905138 [hep-th] .

[7] K.-M. Lee and P. Yi, Phys.Rev. D56 (1997) 3711, arXiv: hep-th/9702107 [hep-th] . K.-M. Lee, Phys.Lett. B426 (1998) 323, arXiv: hep-th/9802012 [hep-th] . K.-M. Lee and C.-H. Lu, Phys.Rev. D58 (1998) 025011, arXiv: hep-th/9802108 [hep-th] .

[8] T. C. Kraan and P. van Baal, Phys.Lett. B428 (1998) 268, arXiv: hep-th/9802049 [hep-th] ; Nucl.Phys. B533 (1998) 627, arXiv: hep-th/9805168 [hep-th] .

[9] T. C. Kraan and P. van Baal, Phys.Lett. B435 (1998) 389, arXiv : hep-th/9806034 [hep-th].

[10] M. N. Chernodub, T. C. Kraan, and P. van Baal, Nucl. Phys. Proc. Suppl. 83 (2000) 556, arXiv:hep-lat/9907001.

[11] P. van Baal and A. Wipf, Phys.Lett. B515 (2001) 181, arXiv: hep-th/0105141 [hep-th] . F. Bruckmann and P. van Baal, Nucl.Phys. B645 (2002) 105, arXiv: hep-th/ 0209010 [hep-th ] . E.-M. Ilgenfritz, M. Müller-Preussker, B. V. Martemyanov, and P. van Baal, Phys. Rev. D69 (2004) 097901, arXiv: hep-lat/ 040202 0. F. Bruckmann, D. Nogradi, and P. van Baal, Nucl.Phys. B698 (2004) 233, arXiv: hep-th/0404210 [hep-th] . 
[12] F. Bruckmann, E.-M. Ilgenfritz, B. V. Martemyanov, and P. van Baal, Phys.Rev. D70 (2004) 105013, arXiv:hep-lat/0408004 [hep-lat].

[13] P. van Baal, Proceedings Lattice fermions and structure of the vacuum, JINR Dubna, (1999) 269, arXiv:hep-th/9912035 [hep-th].

[14] F. Bruckmann, E.-M. Ilgenfritz, B. V. Martemyanov, M. Müller-Preussker, D. Nogradi, D. Peschka, and P. van Baal, Nucl. Phys. Proc. Suppl. 140 (2005) 635, arXiv : hep-lat / 0408036.

F. Bruckmann, D. Nogradi, and P. van Baal, Few Body Syst. 36 (2005) 5.

[15] P. van Baal, A course in field theory. Kindle Edition, 2014.

[16] G. 't Hooft and C. P. Korthals Altes, Taming the Forces Between Quarks and Gluons - Calorons Out of The Box, selected papers by P. van Baal. World Scientific, 2013.

[17] A. Belavin, A. M. Polyakov, A. Schwartz, and Y. Tyupkin, Phys.Lett. B59 (1975) 85.

[18] G. 't Hooft, Phys.Rev. D14 (1976) 3432. C. G. Callan, Jr., R. F. Dashen, and D. J. Gross, Phys.Rev. D17 (1978) 2717; Phys.Rev. D19 (1979) 1826.

H. Levine and L. G. Yaffe, Phys.Rev. D19 (1979) 1225.

[19] A. Jevicki, Phys. Rev. D21 (1980) 992. E.-M. Ilgenfritz and M. Müller-Preussker, Nucl.Phys. B184 (1981) 443; Phys.Lett. B99 (1981) 128. G. Münster, Z.Phys. C12 (1982) 43.

E. V. Shuryak, Nucl.Phys. B203 (1982) 93; Nucl.Phys. B203 (1982) 116.

D. Diakonov and V. Y. Petrov, Nucl.Phys. B245 (1984) 259.

[20] S. L. Adler, Phys.Rev. 177 (1969) 2426. J. Bell and R. Jackiw, Nuovo Cim. A60 (1969) 47. W. A. Bardeen, Nucl.Phys. B75 (1974) 246.

[21] M. Atiyah and I. Singer, Annals Math. 93 (1971) 139; Proc.Nat.Acad.Sci. 81 (1984) 2597.

[22] R. Crewther, "Chirality selection rules and the U(1) problem,” Phys.Lett. B70 (1977) 349.

[23] L. Giusti, G. C. Rossi, M. Testa, and G. Veneziano, Nucl.Phys. B628 (2002) 234, arXiv:hep-lat/0108009 [hep-lat].

[24] L. Giusti, G. C. Rossi, and M. Testa, Phys.Lett. B587 (2004) 157, arXiv: hep-lat / 0402027 [hep-lat].

[25] E. Witten, Nucl.Phys. B156 (1979) 269.

[26] G. Veneziano, Nucl.Phys. B159 (1979) 213-224.

[27] T. Schäfer and E. V. Shuryak, Rev.Mod.Phys. 70 (1998) 323, arXiv : hep-ph/ 9610451 [hep-ph].

[28] D. Diakonov, Prog.Part.Nucl.Phys. 51 (2003) 173, arXiv: hep-ph/0212026 [hep-ph]

[29] D. J. Gross, R. D. Pisarski, and L. G. Yaffe, Rev.Mod.Phys. 53 (1981) 43.

[30] B. J. Harrington and H. K. Shepard, Phys.Rev. D17 (1978) 2122.

[31] F. Bruckmann, M. García Pérez, D. Nogradi, and P. van Baal, Nucl.Phys.Proc.Suppl. 129 (2004) 727-729, arXiv: hep-lat/0308017 [hep-lat] .

[32] E.-M. Ilgenfritz, B. V. Martemyanov, M. Müller-Preussker, S. Shcheredin, and A. I. Veselov, Phys.Rev. D66 (2002) 074503, arXiv: hep-lat/0206004 [hep-lat]. 
[33] E.-M. Ilgenfritz, B. V. Martemyanov, M. Müller-Preussker, and A. I. Veselov, Phys.Rev. D69 (2004) 114505, arXiv:hep-lat/0402010 [hep-lat]; Phys.Rev. D71 (2005) 034505, arXiv:hep-lat/0412028 [hep-lat]; Phys.Rev. D73 (2006) 094509, arXiv:hep-lat/0602002 [hep-lat]. V. G. Bornyakov, E.-M. Ilgenfritz, B. V. Martemyanov, S. M. Morozov, M. Müller-Preussker, and A. I. Veselov, Phys.Rev. D76 (2007) 054505, arXiv:0706.4206 [hep-lat].

[34] V. G. Bornyakov, E.-M. Ilgenfritz, B. V. Martemyanov, and M. Müller-Preussker, Phys.Rev. D79 (2009) 034506, arXiv:0809.2142 [hep-lat].

[35] F. Bruckmann, E.-M. Ilgenfritz, B. V. Martemyanov, and B. Zhang, Phys. Rev. D81 (2010) 074501, arXiv:0912.4186 [hep-th] .

[36] E.-M. Ilgenfritz, M. Müller-Preussker, and D. Peschka, Phys.Rev. D71 (2005) 116003, arXiv:hep-lat/050 3020 [hep-lat].

[37] E.-M. Ilgenfritz, B. V. Martemyanov, and M. Müller-Preussker, Phys.Rev. D89 (2014) 054503, arXiv:1309.7850 [hep-lat]. V. G. Bornyakov, E.-M. Ilgenfritz, B. V. Martemyanov, and M. Müller-Preussker, arXiv:1410.4632 [hep-lat].

[38] A. Belavin, V. Fateev, A. S. Schwarz, and Y. Tyupkin, Phys.Lett. B83 (1979) 317.

[39] V. A. Fateev, I. V. Frolov, and A. S. Shvarts, Nucl.Phys. B154 (1979) 1.

[40] P. Gerhold, E.-M. Ilgenfritz, and M. Müller-Preussker, Nucl.Phys. B760 (2007) 1, arXiv:hep-ph/0607315 [hep-ph]. D. Diakonov, N. Gromov, V. Petrov, and S. Slizovskiy, Phys.Rev. D70 (2004) 036003, arXiv: hep-th/0404042 [hep-th] . D. Diakonov and N. Gromov, Phys. Rev. D72 (2005) 025003, arXiv: hep-th/0502132. D. Diakonov and V. Y. Petrov, Phys.Rev. D76 (2007) 056001, arXiv:0704.3181 [hep-th]; AIP Conf. Proc. 1134 (2009) 190, arXiv:0809.2063 [hep-th] . F. Bruckmann, S. Dinter, E.-M. Ilgenfritz, B. Maier, M. Müller-Preussker, and M. Wagner, Phys.Rev. D85 (2012) 034502, arXiv:1111.3158 [hep-ph]. E. V. Shuryak and T. Sulejmanpasic, Phys.Rev. D86 (2012) 036001, arXiv: 1201.5624 [hep-ph]; Phys.Lett. B726 (2013) 257, arXiv: 1305.0796 [hep-ph] . P. Faccioli and E. V. Shuryak, Phys.Rev. D87 (2013) 074009, arXiv: 1301.2523 [hep-ph]. R. Larsen and E. V. Shuryak, arXiv:1408.6563 [hep-ph] .

[41] J. Greensite, Eur.Phys.J.ST 140 (2007) 1-52; Lect.Notes Phys. 821 (2011) 1-211.

[42] P. C. Argyres and M. Ünsal, JHEP 1208 (2012) 063, arXiv:1206.1890 [hep-th] . E. Poppitz, T. Schäfer, and M. Ünsal, JHEP 1303 (2013) 087, a rXiv : 1212 . 1238. G. V. Dunne and M. Ünsal, Phys.Rev. D89 (2014) 105009, arXiv: 1401.5202 [hep-th] .

[43] P. Di Vecchia, K. Fabricius, G. Rossi, and G. Veneziano, Nucl.Phys. B192 (1981) 392.

N. Makhaldiani and M. Müller-Preussker, JETP Lett. 37 (1983) 523.

K. Fabricius and G. Rossi, Phys.Lett. B127 (1983) 229.

[44] B. Alles, M. Campostrini, A. Di Giacomo, Y. Gunduc, and E. Vicari, Phys.Rev. D48 (1993) 2284, arXiv:hep-lat/9302004 [hep-lat]. B. Alles, M. D'Elia, and A. Di Giacomo, Nucl.Phys. B494 (1997) 281, arXiv: hep-lat/9605013 [hep-lat].

[45] B. Berg, Phys.Lett. B104 (1981) 475. S. Itoh, Y. Iwasaki, and T. Yoshie, Phys.Lett. B147 (1984) 141. M. Teper, Phys.Lett. B171 (1986) 86. E.-M. Ilgenfritz, M. Laursen, G. Schierholz, M. Müller-Preussker, and H. Schiller, Nucl.Phys. B268 (1986) 693.

[46] M. Falcioni, M. Paciello, G. Parisi, and B. Taglienti, Nucl.Phys. B251 (1985) 624. APE Collaboration, M. Albanese et al., Phys.Lett. B192 (1987) 163-169. 
[47] C. Morningstar and M. J. Peardon, Phys. Rev. D69 (2004) 054501, arXiv: hep-lat/ 0311018.

[48] A. Hasenfratz and F. Knechtli, Phys.Rev. D64 (2001) 034504, arXiv: hep-lat/ 0103029 [hep-lat].

[49] T. A. DeGrand, A. Hasenfratz, and D.-c. Zhu, Nucl.Phys. B475 (1996) 321, arXiv:hep-lat/9603015 [hep-lat]. M. Feurstein, E.-M. Ilgenfritz, M. Müller-Preussker, and S. Thurner, Nucl.Phys. B511 (1998) 421, arXiv: hep-lat/9611024 [hep-lat] . T. A. DeGrand, A. Hasenfratz, and T. G. Kovacs, Nucl.Phys. B505 (1997) 417, arXiv:hep-lat/9705009 [hep-lat]. T. A. DeGrand, A. Hasenfratz, and T. G. Kovacs, Nucl.Phys. B520 (1998) 301, arXiv: hep-lat/9711032 [hep-lat ]. A. Hasenfratz and C. Nieter, Phys.Lett. B439 (1998) 366, arXiv:hep-lat/9806026 [hep-lat] .

[50] M. Lüscher, Commun.Math.Phys. 293 (2010) 899, arXiv:0907.5491 [hep-lat]; JHEP 1008 (2010) 071, arXiv: 1006.4518 [hep-lat]; JHEP 1304 (2013) 123, arXiv:1302.5246 [hep-lat]; JHEP 1406 (2014) 105, arXiv: 1404.5930 [hep-lat].

[51] M. Lüscher, Commun.Math.Phys. 85 (1982) 39.

[52] P. Woit, Phys.Rev.Lett. 51 (1983) 638.

[53] A. Phillips and D. Stone, Commun.Math.Phys. 103 (1986) 599.

[54] P. H. Ginsparg and K. G. Wilson, Phys.Rev. D25 (1982) 2649.

[55] P. Hasenfratz, V. Laliena, and F. Niedermayer, Phys. Lett. B427 (1998) 125, arXiv:hep-lat/9801021.

[56] M. Lüscher, Phys.Lett. B428 (1998) 342, arXiv:hep-lat/9802011 [hep-lat].

[57] H. Neuberger, Phys. Lett. B417 (1998) 141-144, arXiv: hep-lat/9707022; Phys. Lett. B427 (1998) 353-355, arXiv: hep-lat/9801031.

[58] D. B. Kaplan, Phys.Lett. B288 (1992) 342, arXiv:hep-lat/9206013 [hep-lat]. Y. Shamir, Nucl.Phys. B406 (1993) 90, arXiv: hep-lat/9303005 [hep-lat] .

[59] F. Bruckmann, C. Gattringer, E.-M. Ilgenfritz, M. Müller-Preussker, A. Schäfer, and S. Solbrig, Eur.Phys.J. A33 (2007) 333-338, arXiv: hep-lat/0612024 [hep-lat]. F. Bruckmann, F. Gruber, C. Lang, M. Limmer, T. Maurer, A. Schäfer, and S. Solbrig, PoS CONFINEMENT8 (2008) 045, arXiv: 0901.2286 [hep-lat]. E.-M. Ilgenfritz, D. Leinweber, P. Moran, K. Koller, G. Schierholz, and V. Weinberg, Phys.Rev. D77 (2008) 074502, arXiv: 0801.1725 [hep-lat].

[60] I. Horvath, S. Dong, T. Draper, F. Lee, K. Liu, et al., Phys.Rev. D68 (2003) 114505 , arXiv:hep-lat/0302009 [hep-lat]. I. Horvath, A. Alexandru, J. Zhang, Y. Chen, S. Dong, et al., Phys.Lett. B612 (2005) 21, arXiv: hep-lat/ 0501025 [hep-lat ] . E.-M. Ilgenfritz, K. Koller, Y. Koma, G. Schierholz, T. Streuer, and V. Weinberg, Phys.Rev. D76 (2007) 034506, arXiv:0705.0018 [hep-lat].

[61] J. Smit and J. C. Vink, Nucl.Phys. B286 (1987) 485.

[62] R. G. Edwards, U. M. Heller, and R. Narayanan, Nucl.Phys. B535 (1998) 403, arXiv:hep-lat/9802016 [hep-lat].

[63] M. Lüscher, Phys.Lett. B593 (2004) 296, arXiv: hep-th/0404034 [hep-th] .

[64] L. Giusti and M. Lüscher, JHEP 0903 (2009) 013, arXiv: 0812 . 3638 [hep-lat ]. 
[65] M. Lüscher and F. Palombi, JHEP 1009 (2010) 110, arXiv:1008.0732 [hep-lat ].

[66] K. Cichy, E. Garcia-Ramos, and K. Jansen, JHEP 1310 (2013) 175, arXiv : 1303.1954 [hep-lat].

[67] ETM Collaboration, K. Cichy, E. Garcia-Ramos, and K. Jansen, JHEP 1402 (2014) 119, arXiv:1312.5161 [hep-lat].

[68] M. García Pérez, A. González-Arroyo, A. Montero, and P. van Baal, JHEP 9906 (1999) 001, arXiv:hep-lat/9903022 [hep-lat].

[69] P. de Forcrand, M. García Pérez, and I.-O. Stamatescu, Nucl.Phys. B499 (1997) 409, arXiv:hep-lat/9701012 [hep-lat].

[70] S. O. Bilson-Thompson, D. B. Leinweber, and A. G. Williams, Annals Phys. 304 (2003) 1, arXiv:hep-lat/0203008 [hep-lat].

[71] V. G. Bornyakov, E.-M. Ilgenfritz, B. V. Martemyanov, V. K. Mitrjushkin, and M. Müller-Preussker, Phys.Rev. D87 (2013) 114508, arXiv:1304.0935 [hep-lat] .

[72] M. I. Buchoff, M. Cheng, N. H. Christ, H. T. Ding, C. Jung, et al., Phys.Rev. D89 (2014) 054514, arXiv:1309.4149 [hep-lat]. S. Sharma, V. Dick, F. Karsch, E. Laermann, and S. Mukherjee, PoS LATTICE2013 (2014) 164, arXiv:1311.3943 [hep-lat ] . S. Aoki, H. Fukaya, and Y. Taniguchi, Phys.Rev. D86 (2012) 114512, arXiv:1209.2061 [hep-lat]. G. Cossu, S. Aoki, H. Fukaya, S. Hashimoto, T. Kaneko, et al., Phys.Rev. D87 (2013) 114514, arXiv:1304.6145 [hep-lat]. B. B. Brandt, A. Francis, H. B. Meyer, O. Philipsen, and H. Wittig, PoS LATTICE2013 (2014) 162, arXiv: 1310.8326 [hep-lat] .

[73] M. Lüscher and P. Weisz, JHEP 1102 (2011) 051, arXiv:1101.0963 [hep-th].

[74] M. Lüscher, PoS LATTICE2010 (2010) 015, arXiv: 1009.5877 [hep-lat ]; PoS LATTICE2013 (2014) 016, arXiv: 1308.5598 [hep-lat ] .

[75] C. Bonati and M. D'Elia, Phys.Rev. D89 (2014) 105005, arXiv:1401.2441 [hep-lat].

[76] A. González-Arroyo and M. Okawa, arXiv:1410.7862 [hep-lat].

[77] A. Chowdhury, A. K. De, S. De Sarkar, A. Harindranath, S. Mondal, et al., Phys.Lett. B707 (2012) 228, arXiv:1110.6013 [hep-lat].

[78] A. Chowdhury, A. K. De, A. Harindranath, J. Maiti, and S. Mondal, JHEP 1211 (2012) 029, arXiv:1208.4235 [hep-lat].

[79] SESAM, T(X)L Collaboration, G. S. Bali et al., Phys.Rev. D64 (2001) 054502, arXiv:hep-lat/0102002 [hep-lat].

[80] ALPHA Collaboration, M. Bruno, S. Schaefer, and R. Sommer, JHEP 1408 (2014) 150, arXiv:1406.5363 [hep-lat].

[81] A. Chowdhury, A. K. De, A. De Sarkar, S. Harindranath, J. Maiti, et al., Comput.Phys.Commun. 184 (2013) 1439, arXiv:1209.3915 [hep-lat].

[82] A. Ramos and S. Sint, arXiv:1411.6706 [hep-lat].

[83] Z. Fodor, K. Holland, J. Kuti, S. Mondal, D. Nogradi, et al., arXiv:1410.8801 [hep-lat].

[84] L. Del Debbio, L. Giusti, and C. Pica, Phys.Rev.Lett. 94 (2005) 032003, arXiv:hep-th/0407052 [hep-th].

[85] K. Cichy, E. Garcia-Ramos, and K. Jansen, arXiv:1412.0456 [hep-1at]. 
[86] C. Alexandrou, M. Constantinou, T. Korzec, H. Panagopoulos, and F. Stylianou, Phys.Rev. D86 (2012) 014505, arXiv:1201.5025 [hep-lat]. K. Cichy, K. Jansen, and P. Korcyl, Nucl.Phys. B865 (2012) 268, arXiv:1207.0628 [hep-lat].

[87] R. Sommer, Nucl.Phys. B411 (1994) 839, arXiv:hep-lat/9310022 [hep-lat].

[88] ETM Collaboration, K. Cichy, A. Dromard, E. Garcia-Ramos, K. Ottnad, C. Urbach, et al., PoS LATTICE2014 (2014) 075, arXiv:1411.1205 [hep-lat] .

[89] ETM Collaboration, C. Michael, K. Ottnad, and C. Urbach, Phys.Rev.Lett. 111 (2013) 181602, arXiv:1310.1207 [hep-lat].

[90] ETM Collaboration, P. Boucaud et al., Comput.Phys.Commun. 179 (2008) 695, arXiv:0803.0224 [hep-lat]. ETM Collaboration, K. Jansen, C. Michael, and C. Urbach, Eur.Phys.J. C58 (2008) 261, arXiv:0804.3871 [hep-lat].

[91] A. Chowdhury, A. Harindranath, J. Maiti, and P. Majumdar, JHEP 1402 (2014) 045, arXiv:1311.6599 [hep-lat].

[92] M. Creutz, Annals Phys. 339 (2013) 560, arXiv:1306.1245 [hep-lat] .

[93] E.-M. Ilgenfritz and A. Maas, Phys.Rev. D86 (2012) 114508, arXiv:1210.5963 [hep-lat].

[94] F. Bruckmann, P. Buividovich, and T. Sulejmanpasic, Phys.Rev. D88 (2013) 045009 , arXiv:1303.1710 [hep-th] . G. Bali, F. Bruckmann, G. Endrödi, Z. Fodor, S. Katz, et al., JHEP 1404 (2014) 129, arXiv: 1401.4141 [hep-lat] . 